\title{
Health Behaviors and Quality of Life Among Colorectal Cancer Survivors
}

\author{
Elizabeth A. Rohan, PhD, MSW; Julie S. Townsend, MS; Temeika L. Fairley, PhD; and Sherri L. Stewart, PhD
}

\begin{abstract}
Purpose: To examine, at the population level, health behaviors, comorbidities, and health-related quality of life among colorectal cancer (CRC) survivors compared with other cancer survivors and persons without cancer. Methods: We used data from the 2009 and 2010 Behavioral Risk Factor Surveillance System cancer survivor modules. We calculated descriptive statistics, conducted chi-square tests for comparisons, and used multivariable logistic regression analysis to compare CRC survivors with other cancer survivors and persons without cancer. Results: Of the 52,788 cancer survivors included in this analysis, 4001 reported being CRC survivors. When compared with other cancer survivors, CRC survivors reported higher percentages of obesity and lack of physical activity; however, they had lower levels of current smoking. Adjusted results show that CRC survivors were significantly more likely to report lack of physical activity, fair/poor health, and other chronic health conditions compared with persons without a cancer diagnosis. Conversely, CRC survivors reported lower levels of current smoking than persons without cancer. Conclusions: CRC survivors have a higher proportion of heath conditions and behaviors that may significantly increase their risks for recurrence or development of a second cancer. Targeted interventions to address these health issues should be considered. (J Natl Compr Canc Netw 2015;13:297-302)
\end{abstract}

\section{Background}

Colorectal cancer (CRC) is the third most commonly diagnosed cancer and the second leading cause of cancer death among cancers affecting both men and women in the United States. ${ }^{1}$ Advances in early detection and treatment have increased cancer survival ${ }^{2}$ : the overall 5 -year survival rate for those diagnosed with CRC is $64 \%{ }^{3,4}$ In the United States, there are approximately 1.2 million CRC survivors. ${ }^{3}$ As the $\mathrm{CRC}$ survivor population grows, more attention must be paid to their long-term care and follow-up. Clinical studies of cancer survivors have shown that comorbidities, such as obesity, and health behaviors, such as physical inactivity and heavy smoking, greatly influence overall quality of life and long-term survival

\footnotetext{
From the Centers for Disease Control and Prevention, Division of Cancer Prevention and Control, Chamblee, Georgia.

Submitted May 12, 2014; accepted for publication August 4, 2014

The authors have disclosed that they have no financial interests, arrangements, affiliations, or commercial interests with the manufacturers of any products discussed in this article or their competitors. The findings and conclusions in this report are those of the authors and do not necessarily represent the official position of the Centers for Disease Control and Prevention.

Correspondence: Elizabeth A. Rohan, PhD, MSW, Centers for Disease Control and Prevention, Division of Cancer Prevention and Control, Comprehensive Cancer Control Branch, 4770 Buford Highway NE, Chamblee Building 107, MS F76, 4th Floor \#4118.1, Chamblee, GA 30341. E-mail: erohan@cdc.gov
}

of CRC survivors. ${ }^{2,5,6}$ Obesity has been linked to both higher mortality and an increased risk for colon cancer recurrence, ${ }^{7,8}$ and lifetime heavy tobacco use ( $\geq 20$ packyear history) has been shown to negatively affect years of disease-free survival. ${ }^{9}$ Conversely, engaging in physical activity can increase years of disease-free survival. ${ }^{6}$

Several population-based studies have assessed the characteristics of cancer survivors, ${ }^{10-14}$ but few focus primarily on CRC survivors. ${ }^{15}$ This article describes, at the population level, the demographics, comorbid health conditions, health behaviors, health care access, use of preventive health services, and health-related quality of life (HRQOL) of CRC survivors to help public health practitioners and health care clinicians prioritize CRC cancer survivorship interventions. Additionally, this study compared CRC survivors with other cancer survivors and persons without cancer to ascertain whether CRC survivors had unique needs that could be addressed in interventions.

\section{Methods}

Behavioral Risk Factor Surveillance System Survey This study used the Behavioral Risk Factor Surveillance System (BRFSS), a state-based system of health surveys 
Rohan et al

that collects information on health risk behaviors, preventive health practices, and health care access through a cross-sectional, random-digit-dialed telephone survey of noninstitutionalized adults aged 18 years or older, using trained interviewers and standard core and optional questionnaire modules. Surveys are conducted with US state and territorial households each month. ${ }^{16-18}$

Survey respondents were asked if they had ever been told by a doctor, nurse, or other health care professional that they had cancer. If they answered yes, they were asked how many different types of cancer they had, age at diagnosis for their first cancer, and the cancer type (most recently diagnosed cancer site if $>1$ diagnosis was reported). Detailed methodology regarding the cancer survivorship modules has been described elsewhere. ${ }^{11}$ We excluded respondents who had an unknown cancer history or refused to answer the question $(\mathrm{n}=5057)$, or who reported their most recent cancer as nonmelanoma skin cancer $(n=16,497)$.

\section{Data Preparation}

We combined survey years 2009 and 2010 in the final analytic file to increase the CRC survivor sample size. In the 2009 BRFSS survey, cancer survivorship questions were administered by all states, the District of Columbia (DC), and territories. In 2010, 17 states (Alaska, California, Colorado, Connecticut, Indiana, Iowa, Massachusetts, Michigan, Missouri, New Jersey, New Mexico, Ohio, Oklahoma, South Dakota, Texas, Utah, and Wisconsin) and Guam administered the module. For states included in both survey years, survey weights were adjusted to prevent overrepresentation of these states on prevalence estimates. A subset of 23 states was included in the analysis of CRC screening because 8 states in 2009 included CRC screening on their BRFSS questionnaire, but all states, DC, and Guam had CRC screening questions in 2010. Three states had both the 2009 screening questions and the 2010 survivorship module, whereas 5 states included the screening questions in 2009 but did not run the survivorship module in 2010. Therefore, this analysis included 71,720 respondents aged 50 to 75 years who answered questions regarding CRC screening. Similarly, 5 states included questions in 2009 on mammography use, but all states, DC, and territories had them in 2010. This analysis included 39,560 female respondents aged 50 to 74 years from 22 states.

\section{Variables}

We compared CRC survivors with other cancer survivors and respondents without a history of cancer, examining the following demographic characteristics: age at interview, sex, racial/ethnic background, marital status, education level, census region of residence, employment status, health insurance coverage, and inability to see a doctor because of cost. For $\mathrm{CRC}$ and other cancer survivors, we calculated years since diagnosis using the respondents' current age and age at first cancer diagnosis.

We examined the following health behaviors: current smoking status, binge drinking (men having $\geq 5$ and women having $\geq 4$ alcoholic drinks on one occasion in the past month), obesity (body mass index $\geq 30 \mathrm{~kg} / \mathrm{m}^{2}$ ), and no leisure-time physical activity in the past month. We analyzed the following chronic health conditions: cardiovascular disease (CVD); disability from physical, mental, and emotional problems; current asthma; and history of diabetes (not including gestational diabetes). We also examined health care access variables: having a personal health care provider and a routine checkup in the past 2 years. We analyzed receipt of preventive health services: pneumococcal vaccine, influenza vaccine within the past 12 months, recommended CRC screening test, and mammography for breast cancer screening within the past 2 years for women aged 50 to 74 years. The surveillance recommendation for CRC survivors is at 1 and 3 years postoperatively, then every 5 years thereafter, ${ }^{19,20}$ so we restricted our assessment of routine CRC surveillance to CRC survivors (aged 50-75 years) diagnosed 5 or more years ago. For other cancer survivors and persons without cancer, we assessed whether they met US Preventive Services Task Force guidelines for CRC screening. To be considered upto-date, both groups aged 50 to 75 years must have had either a fecal occult blood test (FOBT) within the past year, sigmoidoscopy within the past 5 years and an FOBT in the past 3 years, or colonoscopy within the past 10 years.

The following indicators were used to measure HRQOL ${ }^{21}$ : health status and the CDC Healthy Days measures. As part of the Healthy Days measures, survey respondents were asked how many days during the past 30 days they experienced poor physical and mental health. Respondents who indicated 1 or more days on either question were then asked how many days was their usual activity limited by poor 
physical or mental health. All 3 variables were categorized as either 0 to 13 days or 14 or more days during the past 30 days. Receipt of social support and overall life satisfaction variables were also examined to get a broader picture of the well-being of CRC survivorship.

\section{Statistical Analysis}

SAS Version 9.2 (SAS Institute, Cary, NC) and SAScallable SUDAAN release 10 (RTI International, Research Triangle Park, NC) were used to account for the complex sampling design of the BRFSS and to provide weighted population estimates. ${ }^{18} \mathrm{~A}$ descriptive analysis was first conducted to obtain weighted prevalence estimates with 95\% CIs for demographic characteristics, risk behaviors, chronic medical conditions, preventive medical care, life satisfaction, social support, and HRQOL among CRC survivors, other cancer survivors, and respondents without a history of cancer. $P$ values were calculated using the Wald $F$ test. Comparisons were made directly between CRC survivors and persons without cancer, and between CRC survivors and other cancer survivors.

A multivariable logistic regression was conducted to control for demographic characteristics and health care access on the following outcomes of interest: general health status, receipt of social support, life satisfaction, current smoking status, binge drinking, obesity, no leisure-time physical activity, CVD, disability, current asthma, diabetes, CRC screening, mammography test use, influenza vaccination, pneumococcal vaccination, 14 or more mentally unhealthy days, 14 or more physically unhealthy days, and 14 or more days in which poor health limited usual activities. In each of these models, CRC survivors and other cancer survivors were compared with respondents without a history of cancer (referent group) and adjusted for the following covariates: age, sex, education, race/ethnicity, marital status, health insurance coverage, census region, and employment status. Because more than one outcome for health status and receipt of social support was assessed, multinomial logistic regression was used in these models. The no leisure-time physical activity model also adjusted for activity limitations caused by health problems, and the CRC screening model included having a usual health care provider as a covariate (because this variable is a strong predictor of screening). Health status was also included in the influenza and pneumococcal vaccination models. The mam- mography screening model was adjusted for education, race/ethnicity, marital status, health insurance coverage, employment status, health status, census region, and usual health care provider.

\section{Results}

Of the survey respondents included in this analysis, 436,620 said they had never been diagnosed with cancer, 52,788 reported being survivors of other cancers (primarily breast, prostate, and melanoma), and 4001 said they were CRC survivors (Table 1, available online, in this article, at JNCCN.org). Compared with both other cancer survivors and respondents without cancer, CRC survivors tended to be older and retired, and to have access to health insurance. CRC survivors also tended to be more recently diagnosed than other cancer survivors.

CRC survivors were more likely to be obese than other cancer survivors and respondents without cancer $(29.1 \%$ vs $27.5 \%$ and $26.8 \% ; P=.0003$ and $P<.0001$, respectively) and reported having no leisure-time physical activity $(34.2 \%$ vs $31.0 \%$ and $23.8 \% ; P=.044$ and $P<.0001$, respectively) (Table 2 , available online, in this article, at JNCCN.org). CRC survivors reported being current smokers less often than other cancer survivors and persons without cancer $(11.1 \%$ vs $15.3 \%$ and $17.9 \%$, respectively; $P<.0001)$. CRC survivors more frequently reported having CVD (22.3\% vs $17.9 \%$ and $16.4 \%$, respectively; $P<.0001)$ and diabetes $(20.9 \%$ vs $6.8 \%$ and $8.3 \% ; P=.0002$ and $P<.0001$, respectively) than other cancer survivors and persons without cancer. CRC survivors were more likely to report having a personal health care provider $(95.3 \%$ vs $93.4 \%$ and $80.2 \% ; P=.007$ and $P<.0001$, respectively) and having had a regular checkup more recently $(93.0 \%$ vs $89.6 \%$ and $80.7 \% ; P<.0001)$ than other cancer survivors and persons without cancer, respectively. CRC survivors also more frequently reported being up-to-date with vaccinations for influenza $(61.4 \%$ vs $57.4 \%$ and $36.2 \% ; P<.0001)$ and pneumonia $(54.7 \%$ vs $48.1 \%$ and $20.9 \% ; P=.039$ and $P<.0001$, respectively). CRC survivors were more likely to have undergone mammography screenings than respondents without a history of cancer $(85.1 \%$ vs $78.1 \%$; $P=.031$ ). Among long-term survivors ( $>5$ years postdiagnosis), CRC survivors reported undergoing colonoscopic surveillance according to posttreatment 
Rohan et al

guidelines appropriate for their cancer history more frequently $(87.4 \%)$ than respondents without cancer reported being appropriately screened for CRC (59.4\%; $P<.0001)$.

A higher percentage of CRC survivors reported fair, as opposed to good or excellent, overall health compared with other cancer survivors $(22.3 \%$ vs $19.2 \% ; P<.0001$; Table 3 , available online, in this article, at JNCCN.org). CRC survivors were more likely than other survivors to report 13 or fewer days of poor mental health in the past 30 days $(87.8 \%$ vs $85.2 \% ; P=.022)$. CRC survivors were also more likely than respondents without cancer to report more days of poor physical health $(23.5 \%$ vs $9.8 \%$; $P<.0001)$, including poor health that limited activity $(24.0 \%$ vs $12.3 \% ; P<.0001)$, in the past 30 days. CRC survivors were more likely than respondents without cancer to report "always" receiving needed social support ( $51.4 \%$ vs $48.1 \% ; P=.0003)$.

Adjusted results (Table 4 , available online, in this article, at JNCCN.org) show that CRC survivors were 20\% less likely to be a current smoker (odds ratio [OR], 0.80; 95\% CI, 0.66-0.98) than respondents without cancer. CRC survivors were also $17 \%$ more likely to report not engaging in leisure-time physical activity (OR, 1.17; 95\% CI, 1.01-1.34) than respondents without cancer.

CRC survivors were approximately 30\% more likely to have CVD (OR, 1.35; 95\% CI, 1.17-1.56) or current asthma (OR, 1.31; 95\% CI, 1.03-1.65), or to have ever been told they had diabetes (OR, 1.33; 95\% CI, 1.14-1.56) than respondents without cancer. CRC survivors were also $76 \%$ more likely to report having activity limitations than respondents without cancer (OR, 1.76; 95\% CI, 1.53-2.03). CRC survivors were more likely to receive mammography and vaccinations for influenza and pneumonia compared with respondents without cancer, and were 2.7 times more likely than other cancer survivors to be up-to-date with CRC screening than other cancer survivors (OR, 2.71; 95\% CI, 1.44-5.10).

CRC survivors were more likely than respondents without cancer to be in fair/poor or good health versus excellent/very good health (OR, 3.02; 95\% CI, 2.58-3.53, and OR, 1.95, 95\% CI, 1.662.29 , respectively). CRC survivors were 1.5 times as likely to report being dissatisfied/very dissatisfied versus satisfied/very satisfied with life (OR, 1.47; 95\% CI, 1.05-2.05) compared with respondents with- out cancer. Additionally, CRC survivors were 27\% more likely to have had poor mental health (OR, 1.27 ; $95 \% \mathrm{CI}, 1.05-1.53)$, nearly twice as likely to have had poor physical health (OR, 1.91; 95\% CI, 1.64-2.21), and $62 \%$ more likely to have had activity limitations because of poor health within the past 30 days (OR, 1.62, 95\% CI, 1.34-1.96) compared with respondents without cancer.

The pattern of the adjusted results was similar among CRC survivors and other cancer survivors compared with those without cancer for all variables, except for smoking status. CRC survivors had significantly lower smoking levels (OR, 0.80; 95\% CI, 0.66-0.98) than respondents without cancer, whereas those with other cancers have significantly higher smoking levels (OR, 1.09; 95\% CI, 1.02-1.15).

\section{Discussion}

The adjusted results show that CRC survivors were not significantly different from other cancer survivors, but they were significantly different from respondents without cancer. Specifically, CRC survivors had more comorbidities, were less physically active, and had poorer HRQOL than respondents without cancer.

That CRC survivors were more likely to have CVD, current asthma, or a history of diabetes than respondents without cancer is concerning; studies have shown that CRC survivors are less likely to receive recommended care for cardiac and other chronic conditions, ${ }^{22}$ and that noncancer comorbidities have a greater influence on quality of life for long-term ( $>5$ years since diagnosis) CRC survivors than either stage at diagnosis or time since diagnosis. ${ }^{23}$ The findings support the need for improved care of comorbid conditions among CRC survivors and survivors of other cancers.

CRC survivors were less likely to be current smokers than those without cancer, which is important in promoting their long-term survival. However, CRC survivors reported a lack of physical activity. This is an important area for intervention for CRC survivors, especially because physical inactivity is a risk factor for (precancerous) adenomatous colorectal polyps, ${ }^{24}$ other cancers, and other chronic diseases, such as heart disease.

In terms of routine medical care, medical follow-up, and receiving preventive care, such as vaccinations for influenza and pneumococcus, CRC 
survivors reported better outcomes than other cancer survivors and respondents without cancer. This could be because CRC survivors were more recently diagnosed than other cancer survivors in this study and, therefore, more likely to be under close medical supervision. Still, this finding differs from an earlier study of Medicare claims data ${ }^{22}$ that found CRC survivors to be less likely to undergo routine preventive care, such as influenza vaccination, than matched controls without cancer. Because the data reported here are more recent than the Medicare study, this difference may reflect a positive trend in increased use of preventive services by CRC survivors. In support of this, the findings also show that CRC survivors are more likely to be up-to-date on breast cancer screening than those without cancer and are generally receiving appropriate colonoscopy surveillance. These positive health behaviors should continue to be reinforced by health care providers.

The poor health status reported by CRC survivors may be related to surgical interventions, chemotherapy, or radiation performed as part of treatment. The findings regarding poor mental health in CRC survivors are consistent with those of other studies of CRC survivors that report depression as more prevalent in this population. ${ }^{23}$ This was particularly evident for CRC survivors diagnosed less than 5 years ago, who had a higher prevalence of poor health status and more days of poor mental and physical health (data not shown) compared with longer-term CRC survivors.

The negative issues experienced by CRC survivors reported in this study may be addressed by chronic disease self-management, a promising practice being examined in the context of oncology. ${ }^{25}$ Self-management is facilitated by patient-centered survivorship care planning, including the development and use of a survivorship care plan, which is a written, comprehensive treatment summary and plan for follow-up care. ${ }^{26}$ Programs such as the CDC's National Comprehensive Cancer Control Program have prioritized cancer survivorship care and resources. ${ }^{27}$ These programs work within local communities to promote interventions that adequately address the needs of all survivors.

\section{Strengths and Limitations}

This study has several strengths. The BRFSS is a well-established, population-based survey that incorporates activities to help ensure that quality data are collected in a manner usable by public health practitioners. In using BRFSS in this study, we have conducted the largest population-based health behavior analysis of CRC survivors to our knowledge to date. This study also has several limitations. As with all self-reported data, the findings may be limited by recall and social desirability biases. Although BRFSS survey methodology has recently expanded to include cellular telephones, the surveys used for this report were limited to landline telephones (www. cdc.gov/brfss/about/brfss_today.htm). BRFSS also excludes individuals who are incarcerated or hospitalized, or who reside in institutions, such as nursing homes or inpatient hospice units, which may mean that cancer survivors with more advanced disease are not represented.

\section{Conclusions}

CRC survivors' lives are significantly affected by their illness. The findings can help public health professionals and health care practitioners develop tailored information to address the common occurrence of comorbidities, including the negative effects these can have on quality of life, and interventions, such as appropriate physical activity programs, that may help alleviate these effects. This development can occur through a multidisciplinary approach that includes physicians, nurses, social workers, nutritionists, and exercise therapists. The information and interventions developed would likely benefit survivors of other cancers as well.

\section{References}

1. U.S. Cancer Statistics Working Group. United States Cancer Statistics: 1999-2011 Incidence and Mortality Web-based Report. Atlanta: U.S. Department of Health and Human Services, Centers for Disease Control and Prevention and National Cancer Institute; 2014. Available at: www. cdc.gov/uscs. Accessed February 2, 2015

2. Denlinger CS, Barsevick AM. The challenges of colorectal cancer survivorship. J Natl Compr Canc Netw 2009;7:883-893; quiz 894.

3. American Cancer Society. Cancer Treatment and Survivorship Facts \& Figures 2012-2013. Atlanta: American Cancer Society; 2012. Available at: file://C:/Users/green/Downloads/2012+Cancer+Treatment+\%2526+Survi vorship+Facts+\%2526+Figures.pdf. Accessed February 2, 2015.

4. Edwards BK, Ward E, Kohler BA, et al. Annual report to the nation on the status of cancer, 1975-2006, featuring colorectal cancer trends and impact of interventions (risk factors, screening, and treatment) to reduce future rates. Cancer 2010;116:544-573.

5. Meyerhardt JA, Heseltine D, Niedzwiecki D, et al. Impact of physical activity on cancer recurrence and survival in patients with stage III colon cancer: findings from CALGB 89803. J Clin Oncol 2006;24:3535-3541.

6. Meyerhardt JA, Tepper JE, Niedzwiecki D, et al. Impact of body mass index on outcomes and treatment-related toxicity in patients with stage II and III rectal cancer: findings from Intergroup Trial 0114. J Clin Oncol 2004;22:648-657. 
7. Calle EE, Rodriguez C, Walker-Thurmond K, Thun MJ. Overweight, obesity, and mortality from cancer in a prospectively studied cohort of U.S. adults. N Engl J Med 2003;348:1625-1638.

8. Dignam JJ, Polite BN, Yothers G, et al. Body mass index and outcomes in patients who receive adjuvant chemotherapy for colon cancer. J Natl Cancer Inst 2006;98:1647-1654.

9. Jackson NA, Fuchs CS, Niedzwiecki D, et al. The impact of smoking on cancer recurrence and survival in patients with stage III colon cancer: findings from Intergroup trial CALGB 89803 [abstract]. J Clin Oncol 2008;26(Suppl 1):Abstract 4039.

10. Coups EJ, Ostroff JS. A population-based estimate of the prevalence of behavioral risk factors among adult cancer survivors and noncancer controls. Prev Med 2005;40:702-711.

11. Underwood JM, Townsend JS, Stewart SL, et al. Surveillance of demographic characteristics and health behaviors among adult cancer survivors: Behavioral Risk Factor Surveillance System, United States, 2009. MMWR Surveill Summ 2012;61:1-23.

12. Fairley TL, Pollack LA, Moore AR, Smith JL. Addressing cancer survivorship through public health: an update from the Centers for Disease Control and Prevention. J Womens Health (Larchmt) 2009;18:1525-1531.

13. Tai E, Buchanan N, Townsend J, et al. Health status of adolescent and young adult cancer survivors. Cancer 2012;118:4884-4891.

14. White A, Pollack LA, Smith JL, et al. Racial and ethnic differences in health status and health behavior among breast cancer survivorsBehavioral Risk Factor Surveillance System, 2009. J Cancer Surviv 2013;7:93-103.

15. LeMasters T, Madhavan S, Sambamoorthi U, Kurian K. A populationbased study comparing HRQOL among breast, prostate, and colorectal cancer survivors to propensity score matched controls, by cancer type, and gender [published online ahead of print April 19, 2013]. Psychooncology, doi: $10.1002 /$ pon.3288.

16. Gentry E, Kalsbeek W, Hogelin G, et al. The behavioral risk factor surveys: II. Design, methods, and estimates from combined state data. Am J Prev Med 1985;1:9-14.
17. Centers for Disease Control and Prevention. Public health surveillance for behavioral risk factors in a changing environment: recommendations from the Behavioral Risk Factor Surveillance Team. MMWR Recomm Rep 2003;52(RR-9):1-12

18. Centers for Disease Control and Prevention (CDC). Behavioral Risk Factor Surveillance System Survey Data 2009-2010. Available at: http:// www.cdc.gov/brfss/annual_data/2010/overview_10.pdf. Accessed April 14, 2014.

19. Benson AB III, Venook AP, Bekaii-Saab T, et al. NCCN Clinical Practice Guidelines in Oncology: Colon Cancer. Version 2, 2015. Available at: NCCN.org. Accessed February 2, 2015.

20. Benson AB III, Venook AP, Bekaii-Saab T, et al. NCCN Clinical Practice Guidelines in Oncology: Rectal Cancer. Version 2, 2015. Available at: NCCN.org. Accessed February 2, 2015

21. Centers for Disease Control and Prevention. Measuring Healthy Days. Atlanta, Georgia: CDC, November 2000. Available at: http://www.cdc. gov/hrqol/pdfs/mhd.pdf. Accessed February 2, 2014.

22. Earle CC, Neville BA. Under use of necessary care among cancer survivors. Cancer 2004;101:1712-1719.

23. Ramsey SD, Berry K, Moinpour C, et al. Quality of life in long term survivors of colorectal cancer. Am J Gastroenterol 2002;97:1228-1234.

24. Emmons KM, McBride CM, Puleo E, et al. Prevalence and predictors of multiple behavioral risk factors for colon cancer. Prev Med 2005;40:527534.

25. McCorkle R, Ercolano E, Lazenby M, et al. Self-management: enabling and empowering patients living with cancer as a chronic illness. CA Cancer J Clin 2011;61:50-62.

26. Parry C, Kent EE, Forsythe LP, et al. Can't see the forest for the care plan: a call to revisit the context of care planning. J Clin Oncol 2013;31:26512653.

27. Stewart SL, Lakhani N, Brown PM, et al. Gynecologic cancer prevention and control in the National Comprehensive Cancer Control Program: progress, current activities, and future directions. J Womens Health (Larchmt) 2013;22:651-657. 